\title{
Avisos meteorológicos en el Principat d'Andorra
}

https://doi.org/10.31978/639-19-010-0.721

\author{
Guillem Martín Bellido¹ (Guillem_MartinBellido@govern.ad) \\ Carles Miquel García ${ }^{1,2}$ (carles_miquel@govern.ad) \\ Toni Molné Oviedo ${ }^{1}$ (toni_molne@govern.ad)
}

${ }^{1}$ Govern d'Andorra / Oficina de l'Energia i del Canvi Climàtic / Servei meteorològic

${ }^{2}$ Universidad Rovira i Virgili / Departamento de Geografía

\begin{abstract}
RESUMEN
Andorra es un país con un clima de montaña húmedo de latitudes medias, pero con una fuerte influencia mediterránea al sur del país, donde las características son de un clima mediterráneo continental (MiQuel et al., 2014). Una parte del país corresponde a la cuenca fluvial de la Ariège (Francia). Además, el norte del país también limita con esta cuenca atlántica, y a través de collados de formación glacial se intercambian frecuentemente características atmosféricas entre ambas vertientes. La extensión y la intensidad de los fenómenos en las diferentes zonas de Andorra dependen en buena parte de la intensidad de las corrientes atmosféricas y de la altura en la que circulan. Por otra parte, el fuerte gradiente altitudinal (más de 2000 metros de desnivel) hace que la meteorología, en algunas ocasiones, sea muy diferente entre los valles que forman los $468 \mathrm{~km}^{2}$ de territorio.

Con estas condiciones geográficas, la previsión meteorológica toma complejidad, a la vez que requiere precisión, para predecir con la máxima exactitud posible los tipos de meteoros que afectarán las distintas zonas del país. Desde el Servei Meteorològic Nacional se ha elaborado una división de las zonas meteorológicas que no depende de la división administrativa, sino de cadenas de montañas que frenan o aceleran ciertos procesos atmosféricos que afectan a las diferentes parroquias de Andorra, situadas entre los 900 metros y los 2100 metros.
\end{abstract}

PALABRAS CLAVE: avisos meteorológicos; fenómenos extremos; predicción en montaña; aludes; impactos.

\section{INTRODUCCIÓN}

Los avisos meteorológicos en el Principado de Andorra están en fase de evolución tanto a nivel de comunicación como a nivel de la escala de aviso. Este proceso de transformación se está llevando a cabo con la intención de relacionar el nivel de aviso meteorológico con los impactos que producen los fenómenos meteorológicos.

Este nuevo sistema de avisos conducirá al futuro Pla d'Avisos Meteorològics del Principat d'Andorra. Actualmente, este plan, después de haberlo diseñado en el año 2015, está desde 2016 en fase de desarrollo. En esta primera fase se benefician de él el departamento de Protección Civil, otros organismos gubernamentales de Andorra y algunas empresas con participación pública y de interés para el bienestar y la seguridad de la población, así como los servicios meteorológicos vecinos como son Météo France, AEMET y Meteocat. El plan pasará a una segunda fase cuando se apruebe definitivamente y cuando el Servei Meteorològic Nacional tenga efectivos suficientes para poder implementarlo. En esta segunda fase, además de dar servicio 
al departamento de Protección Civil cada día del año, el conjunto de la población se beneficiará de este tipo de avisos a través de los canales de comunicación del Servei Meteorològic Nacional. Durante la primera fase, los avisos se realizan para las siguientes 24 horas. Durante la segunda fase los avisos se realizarán para las siguientes 48 horas en un primer momento y se trabajará para ampliarlos a 72 horas de previsión. En Andorra, los avisos meteorológicos son los fenómenos de intensidad de lluvia y tormenta, acumulación de lluvia, acumulación de nieve, viento, temperaturas extremas y como aviso especial, aludes.

\section{PROCESO DE ELABORACIÓN DEL PLA D’AVISOS METEOROLÒGICS DEL PRINCIPAT D'ANDORRA}

Aunque el Principado de Andorra es un país pequeño $\left(468 \mathrm{~km}^{2}\right)$ y habrá veces que será necesario establecer un mismo nivel de aviso para todo el territorio, tiene un fuerte gradiente altitudinal y también latitudinal. Se han distinguido 3 zonas: norte, centro y sur (figura 1). Al ser las zonas tan pequeñas, la zona centro se ha creado para que sea una zona que sirva para alargar o limitar latitudinalmente el aviso de la zona norte o la sur, es decir que no es una zona independiente. Según el criterio del predictor y de los umbrales meteorológicos de los diferentes fenómenos definidos para las distintas zonas, tomará el aviso de la zona norte o de la zona sur (MARTín, 2017).

Para dividir el país en zonas meteorológicas se han estudiado las características meteorológicas de cada zona, y la afectación de los diferentes meteoros. Primero se ha estudiado la distribución de estos, y luego el impacto de cada uno de ellos en las distintas zonas desde el inicio de las medidas meteorológicas el año 1934 por parte de FHASA (Forces Hidroelèctriques d'Andorra S.A., actualmente FEDA: Forces elèctriques d'Andorra).

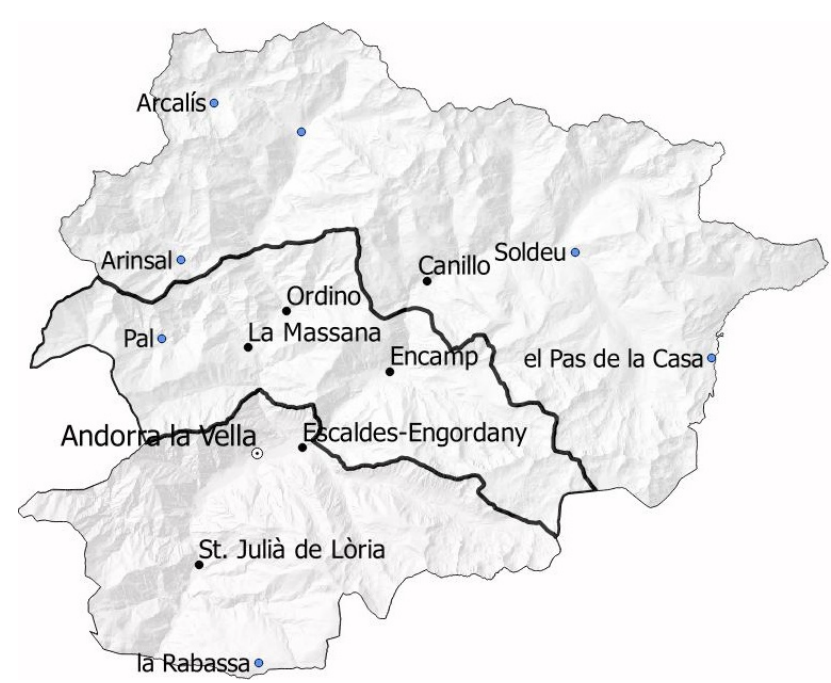

Figura 1. Zonas meteorológicas de Andorra. Fuente: Guillem Martín.

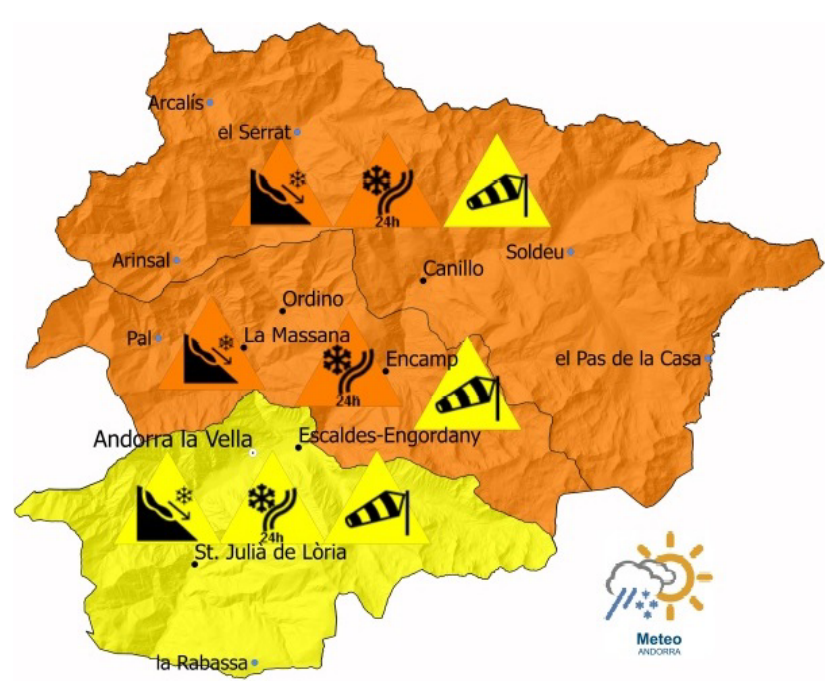

Figura 2. Ejemplo de mapa de aviso meteorológico de Andorra.

\section{CARACTERÍSTICAS DE LAS DISTINTAS ZONAS Y DISTINCIÓN DE FENÓMENOS}

Como los fenómenos meteorológicos y sus impactos están ligados a la orografía del terreno, para delimitar las zonas meteorológicas de Andorra se ha utilizado el esquema de reconstrucción del máximo glacial en el Principado de Andorra durante el máximo glacial que ocurrió durante la glaciación de Würm (figura 3), hace unos 20000 años y que modeló los valles del Principado como los conocemos hoy en día (CopOns, 2012). 


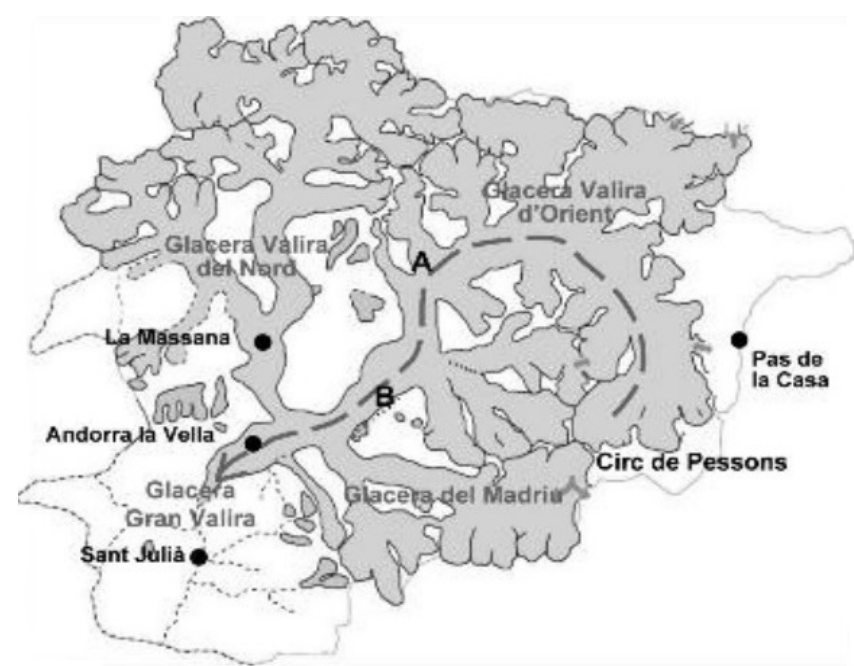

Figura 3. Extensión de los glaciares en su máximo glacial de la glaciación de Würm.

Fuente: Ramon Copons.

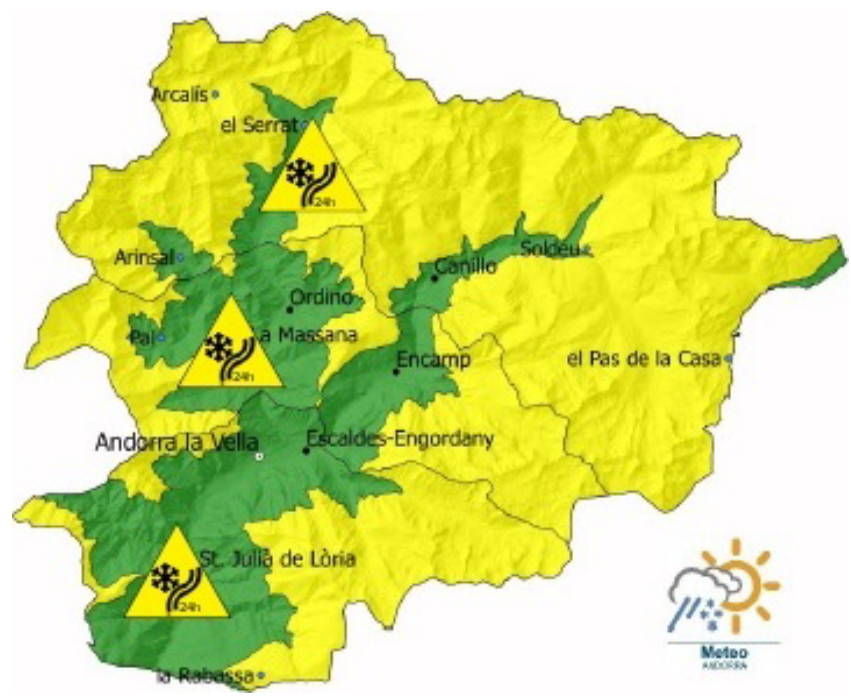

Figura 4. Ejemplo de aviso meteorológico altitudinal por acumulación de nieve. Fuente: Guillem Martín.

Por otra parte, los distintos fenómenos meteorológicos pueden ser avisados de distinta forma. Se pueden emitir avisos por zonas y/o altitudinales (figura 4). Por ejemplo, en situación anticiclónica con circulación de norte se pueden formar precipitaciones orográficas que afecten solamente la zona norte, pero el viento se tendrá que avisar en todo el país. O bajo la influencia de la corriente en chorro en altura, será necesario un aviso por viento solo a partir de, por ejemplo, 2200 metros.

Así, los avisos por acumulación de nieve se pueden emitir por zonas y por altitud; los avisos de viento se pueden emitir por el conjunto del país y por altitud; los de acumulación de precipitación se pueden emitir por zonas; los de intensidad y tormenta por el conjunto del país; los de temperaturas extremas por zonas; y los aludes por zonas y por altitud.

La zona norte está caracterizada por una mayor altitud media y por unos valles más suavizados por la acción glacial de alta montaña. Los valles principales del norte del país (Comapedrosa, Tristaina y Valira d'Orient) están orientados aproximadamente de oeste a este, pero tienen numerosas entradas de valles secundarios en dirección norte-sur como son los valles del Pla de l'Estany, Rialb, Ransol e Incles. También se tiene que destacar el importante papel que desempeña el valle francés de Soulcem, orientado de norte a sur en la vertiente atlántica, en la meteorología andorrana y que su influencia ha determinado la extensión de la frontera entre la zona norte y la centro. En esta zona, la norte, la altitud mínima son 1340 metros sobre el nivel del mar y la máxima son los más de 2900 metros del pico más alto de Andorra, el Comapedrosa. Esta limita con la Ariège francesa y con la parte más occidental de los Pyrenées Orientales, y es una zona muy afectada por frentes y circulaciones de norte por débiles que sean, gracias a la proximidad geográfica con la vertiente atlántica, pero también gracias a las numerosas comunicaciones entre la vertiente atlántica y la mediterránea que forman los collados de transfluencia glacial de los valles secundarios nombrados.

La zona centro se ve afectada por circulaciones de norte, en cuanto a precipitaciones, cuando estas ya son un poco más intensas ya que los valles que se unen con la zona norte están orientados de norte a sur. A nivel orográfico, las vertientes son más pronunciadas, con lo que el impacto de la intensidad de lluvia es mayor que en la zona norte. En situación de circulación de sur-oeste, con abundantes lluvias, es una zona que recibe mucha precipitación tanto en el área de Setúria (oeste) como en el área del Madriu alto.

La zona sur se ve afectada por circulaciones de norte cuando estas se generalizan y afectan el conjunto del macizo pirenaico. Normalmente, en estas situaciones es pertinente diferenciar el nivel de aviso entre la zona norte y centro de la zona sur. Igualmente, cuando la circulación tiene componente sur es pertinente, la mayoría de veces, diferenciar el nivel de aviso entre la zona sur y centro de la norte. Esta zona se caracteriza por 
montañas más verticales que en el resto de zonas que bajan desde los 2600 metros hasta los 900 en menos de $2 \mathrm{~km}$ de distancia horizontal, por lo que es la zona donde las avenidas de agua tienen mayor impacto. Por otra parte, las precipitaciones en forma de nieve afectan a un mayor número de personas al estar la capital en esta zona, por lo que los umbrales se han tenido que adaptar a los impactos, pero también a la ocurrencia.

En definitiva, las zonas se han definido por su distribución de precipitación en distintos tipos de circulación atmosférica, lo que influye también en la distribución y ocurrencia de los aludes. Por este motivo, la zonificación del boletín de peligro de aludes de Andorra utiliza la misma separación de zonas.

\section{DEFINICIÓN DE LOS UMBRALES}

La definición de los umbrales meteorológicos para cada fenómeno ha tenido en cuenta la ocurrencia del fenómeno y el impacto, con el fin de avisar a la población solo cuando sea necesario y que el nivel de aviso sea lógico con los impactos, es decir, de la vulnerabilidad del territorio. Así pues, dependiendo de las situaciones, el predictor meteorológico podrá elevar o bajar el nivel de aviso si cree que la situación lo merece, como veremos en el siguiente apartado.

Como ejemplo de estudio y definición de los umbrales, tenemos la distribución de las temperaturas máximas extremas que se muestra en el gráfico de la figura 5. En un país de montaña como es Andorra, donde las temperaturas por encima de $\operatorname{los} 30^{\circ} \mathrm{C}$ deberían ser más raras de lo que son actualmente, $\mathrm{y}$ donde las temperaturas inferiores a $0^{\circ} \mathrm{C}$ deberían ser normales, sobre todo en invierno, para no generar un estado permanente de aviso o bien no avisar nunca de determinados fenómenos, se han tenido en cuenta los percentiles de la serie. Así, un aviso por temperaturas extremas de nivel naranja se producirá, generalmente, cuando las temperaturas estén dentro de los percentiles 2 y 98 .

Por otra parte, para definir los umbrales de precipitación se ha tenido en cuenta la rareza de las acumulaciones de precipitación (figura 6) y los impactos que han producido abundantes precipitaciones en 24 horas como las de noviembre de 1982 o la acumulación de precipitación durante 72 horas, como en octubre de 1987. Así pues, los umbrales de precipitación tienen en cuenta las acumulaciones desde 12 horas hasta 72.

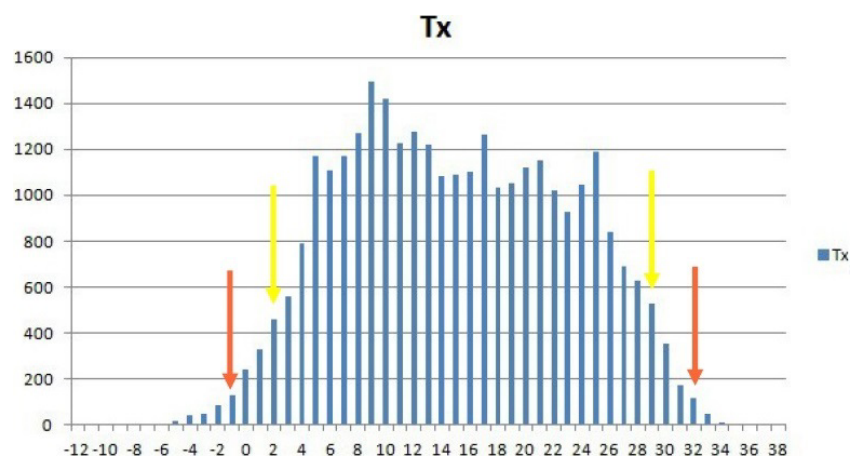

Figura 5. Distribución de las temperaturas máximas a 1130 metros de altura.

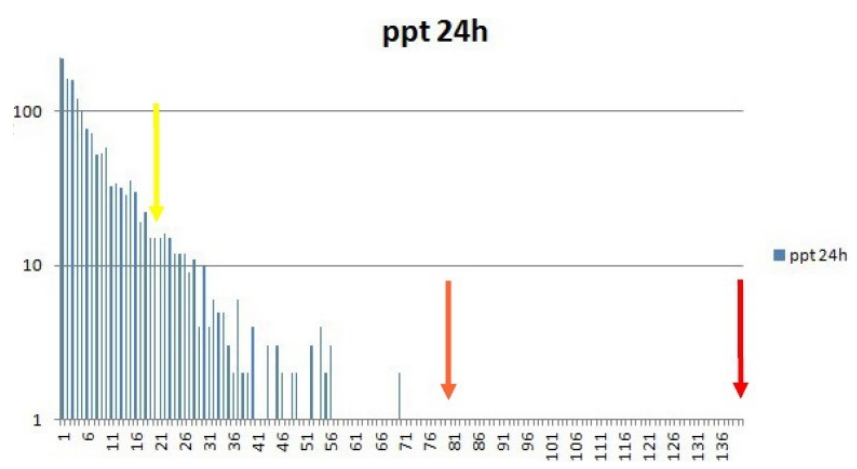

Figura 6. Distribución logarítmica de las acumulaciones de precipitación a 1130 metros de altitud.

Por otra parte, tenemos la intensidad de precipitación, que va unida al aviso de tormenta eléctrica. El umbral de este aviso tiene en cuenta únicamente los impactos, ya que la variabilidad meteorológica de verano no permite establecer una regla lógica para estudiar la rareza de los fenómenos tormentosos.

Para definir todos los umbrales se ha tenido en cuenta la información recibida por Protección Civil sobre los impactos históricos que han producido los distintos fenómenos, así como distintas personas, organizaciones y empresas que explotan instalaciones en alta montaña, como las estaciones de esquí. Por ejemplo, tendría poco sentido emitir un aviso naranja por viento si los remontes siguieran abiertos; o bien la previsión habría sido alarmista o los umbrales no estarían orientados a los impactos y por lo tanto no serían útiles para tomar decisiones preventivas. 


\section{IMPACTOS Y FACTORES QUE LOS INFLUYEN}

Los avisos meteorológicos pueden conllevar activar alertas por parte de Protección Civil, por lo que estos deben ser lo más precisos posibles. El nivel de aviso tiene que conllevar un nivel de alerta de la población adecuado, lógico con la intensidad de los meteoros. Desde los Servicios Meteorológicos Nacionales se tiene que evitar al máximo tanto las falsas alarmas como la omisión de avisos.

Por una parte, desde el Servei Meteorològic Nacional tenemos el principio de no avisar a través del Pla d'avisos meteorològics de fenómenos de intensidad débil, es decir de clasificar como avisos verdes, por ejemplo, lluvias inferiores a $20 \mathrm{~mm}$ en 24 horas o nevadas de $5 \mathrm{~cm}$ a 1500 metros. Pues vivimos en un país de montaña y los ciudadanos tienen que estar acostumbrados a ciertas inclemencias meteorológicas. Los avisos aumentan su intensidad cuando el meteoro empieza a causar cambios en la vida cotidiana de los ciudadanos. Así pues, los umbrales de los distintos meteoros cumplen los siguientes impactos según la intensidad (figura 7).

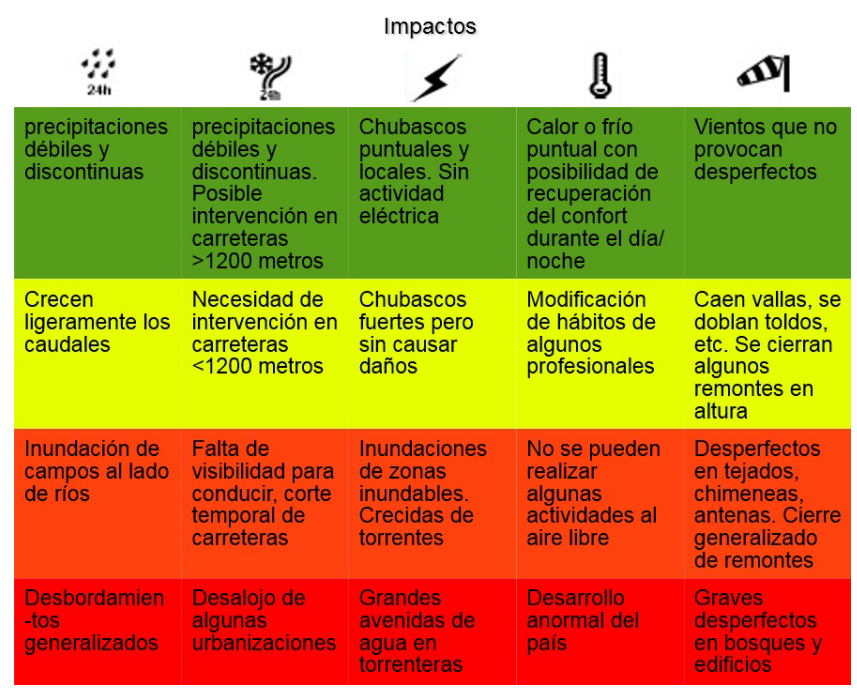

Figura 7. Meteoros e impactos que provocan cuando se registran valores descritos en los umbrales meteorológicos.

Con el objetivo de evitar las falsas alarmas o las omisiones de aviso, los predictores meteorológicos pueden modificar un nivel de aviso si así lo creen conveniente. Algunos ejemplos que pueden modificar el nivel de aviso propuesto según los umbrales son la presencia o no de convergencias de vientos en entornos favorables a la convección, la altura del nivel freático en situaciones de lluvias persistentes, la presencia o no de nieve en el suelo durante olas de frío (la nieve puede aislar del frío las tuberías de agua), o la persistencia de la temperatura máxima durante las olas de calor.

Un claro ejemplo lo tenemos en los impactos que provocaron las olas de frío de febrero de 1956 y la de enero de 1985. Este último episodio, al ser un año con muy poca nieve provocó el congelamiento de tuberías de agua, mientras que en el año 1956, cuando hizo todavía más frío pero había una cobertura extensa de nieve, no hubo ningún problema con las conducciones de agua potable.

\section{CONCLUSIONES}

Orientar los avisos meteorológicos a impactos necesita de un minucioso trabajo de análisis de fenómenos extremos para poder definir los umbrales. Además, como hemos visto en el apartado anterior, la emisión de avisos meteorológicos orientados a impactos necesita una validación o modificación, si es necesario, por parte de un equipo de predictores meteorológicos que conozcan cómo reacciona el territorio a los meteoros previstos.

Los impactos que provocan un meteoro son función de la intensidad de este y de la vulnerabilidad del territorio. La primera variable puede ser prevista por los meteorólogos, mientras la segunda puede ser modificada por fenómenos naturales o por causas antrópicas. Por lo tanto, los umbrales están en constante supervisión.

Algunas veces, como podemos ver en la figura 8, se construyen protecciones frente a meteoros extremos para reducir la vulnerabilidad de una población. En este caso, la construcción de un dique para limitar la expansión de un alud cuando llega al fondo del valle y que no afecte a más viviendas de las que ya están construidas en el mismo canal, es muy efectiva junto a dispositivos de protección en la zona de salida del alud, 
pero esto ¿es motivo suficiente como para modificar al alza un umbral de aviso? La respuesta es que para este fenómeno, no, ya que este impacto estaría clasificado como un nivel de aviso rojo, y esto quiere decir que el impacto provocado por este meteoro (intensas y abundantes nevadas) es muy severo y el peligro puede venir de donde menos lo esperamos, por ejemplo de otro canal de aludes que hay al lado. Aunque es más pequeño puede provocar también un impacto extremo.

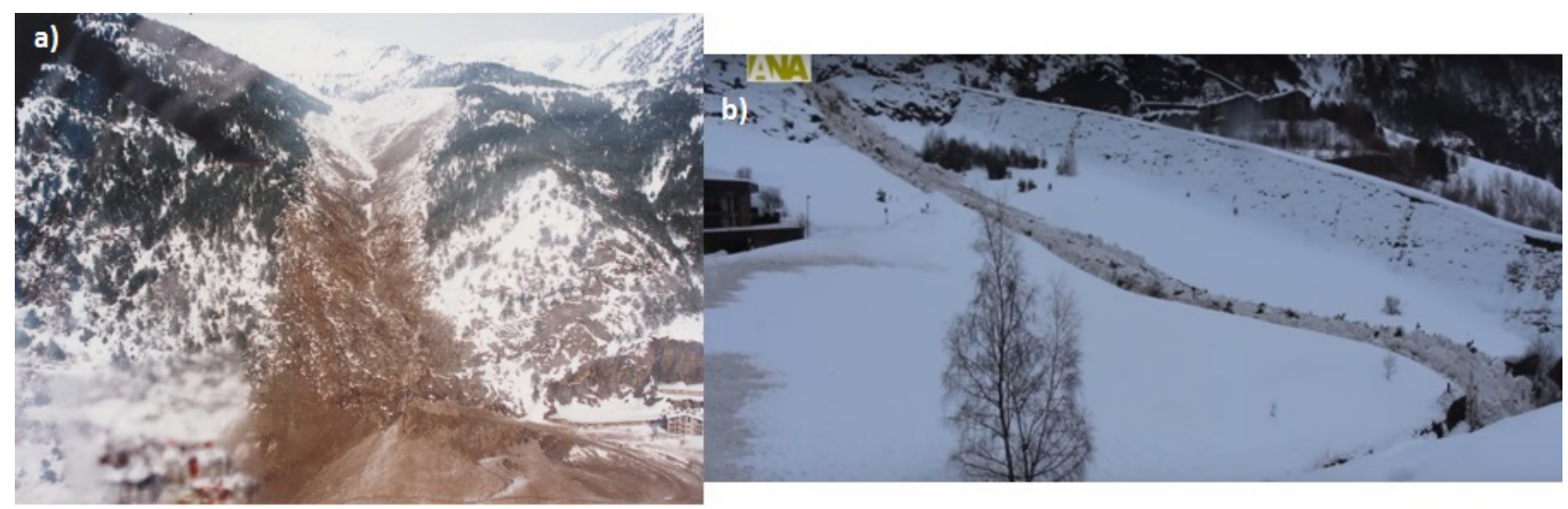

Figura 8. a) El alud del 8 de febrero de 1996 en Arinsal que afectó viviendas y un hotel previamente desalojados. b) El mismo alud el 26 de febrero de 2015 con menor impacto gracias a las medidas de protección. Fotografía: Albert Reyes y Agència de Noticies d'Andorra.

Así pues, la revisión de umbrales orientados a impactos es más viable en avisos de niveles inferiores, avisos de nivel verde, amarillo y naranja.

Pero los Servicios Meteorológicos Nacionales también tenemos que prever la opción de modificar a la baja estos umbrales. Por ejemplo, un incendio forestal o vientos huracanados pueden destruir un bosque y eliminar su función protectora contra las grandes avenidas de agua, deslizamientos de tierra o aludes. O como pasó el 21 de julio de 2015 en Andorra, una fuerte tormenta causó grandes y profundas estrías en las cimas de la montaña de Claror, de donde se recoge agua potable para la población de Escaldes-Engordany. Durante los dos siguientes años, las lluvias, por débiles que eran, provocaban cortes de agua en la segunda ciudad más poblada de Andorra. El impacto era extremo, se tuvieron que modificar los umbrales para esta zona hasta que se implementaron las medidas de adaptación necesarias para recuperar el nivel de vulnerabilidad anterior al episodio de julio de 2015.

\section{AGRADECIMIENTOS}

Agradecer la invitación de la Delegación Territorial de AEMET en Cataluña para participar en este VISimposio Nacional de Predicción y a los Servicios Centrales de AEMET por el cálido acogimiento durante los días del Simposio.

\section{REFERENCIAS}

Copons, R., 2012. Les valls d'Andorra: hereves d'antigues glaceres. Universitat Catalana d'Estiu 2011.

Martín, G., 2017. Més càlid, més sec i més humit. Els llindars d'alerta meteorològica. Universitat Catalana d'Estiu 2016.

Miquel, C., Armengol, J. L., Dobarro, J. y Robira, N., 2014. Biennial Update Report. 